\title{
Neural Activity and Neurotransmission Regulate the Maturation of the Innervation Field of Cortical GABAergic Interneurons in an Age-Dependent Manner
}

\author{
Elie Baho and Graziella Di Cristo \\ Centre Hospitalier Universitaire Sainte-Justine and Université de Montréal, Montréal, Quebec H3T 1C5, Canada
}

\begin{abstract}
Neural activity guides the patterning of neuron synaptic territory in the developing nervous system. Evidence supporting this hypothesis comes from numerous studies on projection neurons in neuromuscular and visual systems. It is unknown whether the innervation field of GABAergic interneurons, which forms local dense innervations, follows similar rules.

Cortical basket cells innervate hundreds of pyramidal cell somata and proximal dendrites. Thanks to this connectivity pattern, they can tightly control neural excitability and synchronization. Here we show that reducing excitation, and thus neurotransmitter release, in mouse cortical single basket cells in slice cultures decreases the number of innervated cells without changing the pattern of perisomatic innervation, both at the peak and after the proliferation phase of perisomatic synapse formation. Conversely, suppressing neurotransmitter release in single basket cells can have completely opposite effects depending on the developmental stage. Our results reveal a remarkably specific and age-dependent role of neural activity and neurotransmission levels in the establishment of the synaptic territory of cortical GABAergic cells.
\end{abstract}

\section{Introduction}

Basket cells, a subtype of GABAergic interneurons, innervate hundreds of postsynaptic targets with multiple synapses clustered around the cell body and proximal dendrites. Their proper functioning is essential for neural network activity, and their dysfunction has been linked to the occurrence of cognitive dysfunctions (Huang et al., 2007; Rossignol, 2011).

The establishment of mature innervation by a single basket cell requires several steps, from finding the right cell target and selecting the appropriate subcellular location for synapse localization to terminal branch formation and synapse proliferation. Recent studies have started to elucidate the mechanisms regulating the maturation of GABAergic innervation. For example, it has been shown that target selection is mainly regulated by molecular cues (Ango et al., 2004, 2008), while synapse proliferation is dependent on neural activity levels and GABAergic neurotransmission (Chattopadhyaya et al., 2004, 2007). Our knowledge of the molecular mechanisms mediating activity-dependent GABAer-

\footnotetext{
Received Aug. 24, 2011; revised Nov. 11, 2011; accepted Nov. 16, 2011.

Author contributions: E.B. and G.D.C. designed research; E.B. performed research; E.B. and G.D.C. analyzed data; E.B. and G.D.C. wrote the paper.

G.D.C. was supported by the Canadian Institutes of Health Research, the National Alliance for Research on Schizophrenia and Depression (Katowitz/Radin Investigator), the Natural Sciences and Engineering Research Council of Canada, the Scottish Rite Charitable Foundation, Canada Foundation for Innovation, and the Canada Research Chair program. We thank Drs. Bidisha Chattopadhyaya, Edward S. Ruthazer, Elsa Rossignol, Z. Josh Huang, and Xiaoyun Wu for critical reviewing of this manuscript. TeNT-Lc::GFP was a kind gift of Dr. Martin P. Meyer (King's College London, UK). AlstR was a kind gift of Drs. Keith K. Murai and Sabrina Chierzi (McGill University, Canada).

Correspondence should be addressed to Graziella Di Cristo, Research Center, CHU Ste-Justine/Université de Montréal 3175, Côte-Ste-Catherine, Montréal, QC H3T 1C5, Canada. E-mail: graziella.dicristo@recherche-stejustine.qc.ca.

DOI:10.1523/JNEUROSCI.4352-11.2012

Copyright $\odot 2012$ the authors $\quad 0270-6474 / 12 / 320911-08 \$ 15.00 / 0$
}

gic synapse proliferation has also improved; indeed, recent studies identified several factors that either stimulate (e.g., BDNF, GAD67, NCAM; for review, see Huang et al., 2007) or inhibit (polysialated-NCAM; Di Cristo et al., 2007) the formation of perisomatic GABAergic innervation. The interplay among these factors most likely determines the appropriate number of GABAergic synapses and the time course of their maturation. All of these studies focus on the mechanisms regulating the number of GABAergic synapses formed by a basket cell around single postsynaptic somata; however, how a basket cell decides the number of target cells to innervate is not well understood.

After decades of study, researchers have shown that neural activity plays an essential role in transforming immature circuits into the organized connections that subserve adult brain function. The essential role of neural activity in the establishment of the neural innervation field has been best studied in the visual system (Huberman et al., 2008), the neuromuscular junction (Buffelli et al., 2003), and the cerebellum (Bosman and Konnerth, 2009). Cortical GABAergic basket cells differ from retinal ganglion cells (RGCs), motoneurons, and climbing fibers in two fundamental aspects. First, they form a dense local axonal arbor, as opposed to long-distance projections to separate targets, and second, they use GABA, which is an inhibitory neurotransmitter. How neural activity levels influence the establishment of a mature basket axonal arbor territory is unclear.

As key mediators of neural activity, neurotransmitters are particularly well suited to couple functional neurotransmission with synaptic morphogenesis and refinement. By using a single-cell genetic approach to either reduce spiking activity, which in turn reduces neurotransmitter release, or suppress neurotransmitter release in single basket cells in mouse cortex, we found that neu- 
rotransmission effects are critically dependent on the maturation state of the basket cell innervation.

\section{Materials and Methods}

Cortical organotypic culture and biolistic transfection. Slice culture preparation was performed as described by Chattopadhyaya et al. (2004) using mice pups of either sex. To activate the allatostatin receptor (AlstR), allatostatin peptide (10 nM, Phoenix Pharmaceuticals) was added with the culture medium during the specified time window. Gene gun transfection was performed as described by Chattopadhyaya et al. (2004). To transfect both GFP/AlstR (green)-expressing and tdTomato (red)expressing basket cells in the same cortical slice, a set of gold particles was coated with $\mathrm{P}_{\mathrm{G} 67}$-AlstR $(30 \mu \mathrm{g})$ and $\mathrm{P}_{\mathrm{G} 67}-\mathrm{GFP}(30 \mu \mathrm{g})$ constructs and another set was coated with $\mathrm{P}_{\mathrm{G} 67}-t d$ Tomato construct $(30 \mu \mathrm{g})$. After two washes with $100 \% \mathrm{EtOH}$, the two sets of gold particles were mixed and used to prepare bullets. In a parallel set of experiments, we transfected separate cortical slices with either $\mathrm{P}_{\mathrm{G} 67}$-AlstR together with $\mathrm{P}_{\mathrm{G} 67}$-GFP or with $\mathrm{P}_{\mathrm{G} 67}-\mathrm{GFP}$ alone, to control that labeling resolution and bouton size were not dependent on the fluorophore used. The results obtained with the two labeling strategies were not statistically different and were pooled together.

Tetanus toxin light-chain (TeNT-Lc)::GFP was a kind gift from Dr. M. Meyer (King's College London, UK) and was then cloned into the $\mathrm{P}_{\mathrm{G}^{-} 7^{-}}$ vector (Chattopadhyaya et al., 2004). TeNT-Lc-EGFP is a fusion protein whose interaction with proteins in the presynaptic terminals could promote a more compact localization of the fusion protein. EGFP labeling, therefore, might not faithfully represent bouton area. To control for this point, we did two sets of experiments. In one set, we transfected slices with either $\mathrm{P}_{\mathrm{G} 67}$-TeNT-Lc-EGFP together with $\mathrm{P}_{\mathrm{G} 7^{-}}$-tdTomato or $\mathrm{P}_{\mathrm{G}_{67}}{ }^{-}$ EGFP alone. In a second set of experiments, different cortical slices were transfected with either $\mathrm{P}_{\mathrm{G} 67}$-TeNT-Lc-GFP or $\mathrm{P}_{\mathrm{G} 67}$-EGFP. The results obtained with these two labeling strategies were not statistically different and were pooled together.

Analysis of basket cell innervation. For each experimental group, we took care to acquire an equal number of basket cells localized in layers $2 / 3$ and 5/6. Confocal images of the basket cell axon arbors were taken in the first $150 \mu \mathrm{m}$ from the basket cell soma using a $63 \times$ glycerol objective (NA 1.4, Leica) and a Leica TCS SPE. Confocal stack size was $116.4 \times$ $116.4 \mu \mathrm{m}$ with an average depth of $40-70 \mu \mathrm{m}$. Analysis of basket cell perisomatic innervation and bouton size was performed as described by Chattopadhyaya et al. (2007). Only innervated cells were included in this analysis.

To quantify the fraction of pyramidal cell somata potentially innervated by a basket cell axon, we divided the number of NeuN-positive neurons contacted by at least one GFP-positive-bouton by the total number of NeuN-positive cells, in a confocal stack taken in the first $150 \mu \mathrm{m}$ from the basket cell soma (at least 3 stacks per basket cell). This measure critically depends from the pyramidal cell density in a confocal stack. We measured pyramidal cells density and found it to be invariant with respect to the different manipulations. In addition, to control for bias versus denser zones of the axonal field, $3-5$ basket cells per experimental group were fully reconstructed and the fraction of innervated neurons was quantified as described above. On average, between 6 and 9 confocal stacks, with a volume of $174.6 \times 174.6 \times 70-150 \mu \mathrm{m}$, were necessary to reconstruct a basket cell.

All data were first averaged per basket cell; thus, statistical analysis was done using the number of basket cells as $n$.

Immunostaining and $p C R E B$ quantification. Immunohistochemistry was performed as described by Chattopadhyaya et al. (2004). The following antibodies were used: NeuN (monoclonal, 1:400, Millipore), GAD65 (monoclonal, 1:1000, Millipore), and pCREB (Ser133) (rabbit, 1:400, Cell Signaling Technology). For pCREB, experiments were run in duplicate and images were all acquired the same day using the identical confocal parameters and a $20 \times$ water-immersion objective (Leica). Z-stacks were acquired with a $5 \mu \mathrm{m}$ step, exported as TIFF files, and analyzed using Image (NIH) software. For each transfected basket cell, pCREBpositive nuclei were outlined and the intensity levels were measured, after background subtraction, for both the transfected basket cell and 5 neighboring cells in the same confocal plane. The ratio of PCREB intensity levels in the transfected versus untransfected neighboring cells was then calculated and compared across different experimental conditions.

\section{Results}

To explore the role of a basket cell's neural activity and neurotransmission on the establishment of its innervation field, we employed two methods: (1) we reduced neuron excitability in single basket cells using allatostatin-mediated activation of the G-protein-coupled Drosophila allatostatin receptor (AlstR), and (2) we suppressed presynaptic release of the neurotransmitter using a targeted expression of TeNT-Lc in single basket cells in mouse cortical organotypic cultures. The basic features of perisomatic innervation of pyramidal cells by basket interneurons develop in organotypic culture (Chattopadhyaya et al., 2004). In particular, perisomatic innervation matures significantly after the second postnatal week in culture and is influenced by alterations in neural activity (Chattopadhyaya et al., 2004).

The activation of AlstRs has been shown to activate endogenous mammalian G-protein-coupled inwardly rectifying $\mathrm{K}^{+}$ (GIRK) channels. Upon binding to its ligand (allatostatin), AlstR/GIRK complexes cause membrane hyperpolarization and consequently a decrease in neural excitability and action potential firing, which in turn reduces neurotransmitter release (Birgül et al., 1999). In acute brain preparations and in vivo, acute application of allatostatin has been shown to reversibly abolish action potential firing in cells expressing AlstRs (Zhou et al., 2009; Marina et al., 2010).

To reduce neural excitability and simultaneously label single basket cell axons and synapses at high resolution, we used a previously characterized promoter region $\left(\mathrm{P}_{\mathrm{G} 67}\right.$; Chattopadhyaya et al., 2004) to express by biolistic transfection either EGFP together with AlstR, or tdTomato alone, in basket interneurons (Fig. $1 A 1)$. We then added allatostatin $(10 \mathrm{~nm})$ in the culture medium for $7 \mathrm{~d}$, which activated AlstR in GFP-expressing basket cells (green) but not in the neighboring tdTomato-expressing ones (red), in organotypic cultures (Figure 1A2,A3). Like other G-protein-coupled receptors, AlstR might be desensitized or internalized with prolonged exposure to its ligand. To check whether long-term application of allatostatin in our culture conditions was still effective in reducing basket cell excitability $7 \mathrm{~d}$ after the onset of exposure to allatostatin, we used pCREB immunofluorescence as an indicator of neural activity. Basket cells expressing AlstR and treated with allatostatin from equivalent postnatal day 17 (EP17; P4 + $13 \mathrm{~d}$ in vitro) to EP24, during the peak of GABAergic synapse proliferation (Chattopadhyaya et al., 2004) and well after GABA action has become inhibitory, showed reduced pCREB expression in their nuclei as compared with neighboring, untransfected cells (Figure $1 B, C$; mean pCREB expression in transfected cell/neighboring untransfected cells, $0.91 \pm 0.09$ for control basket cells; $0.63 \pm 0.07$ for AlstRexpressing basket cells; $n=9$ basket cells for ctrl and $n=12$ for allatostatin-treated basket cells, from at least 6 different slices; Mann-Whitney on ranks, $p<0.05$ ).

Following neural activity and neurotransmission manipulations in single basket cells, we quantified two aspects of basket cell axon innervation, the extent of perisomatic innervation around single somata (terminal branching, perisomatic synapse density and size) and the fraction of innervated somata. We have previously shown (Chattopadhyaya et al., 2004, 2007) that the vast majority of GFP-labeled boutons in our experimental condition most likely represent presynaptic terminals. Allatostatin-mediated reduction of basket cell firing from EP17-24, during the peak of perisomatic synapse proliferation, did not affect perisomatic bouton maturation. AlstR-expressing bas- 
A1 P4-P5 mouse pup
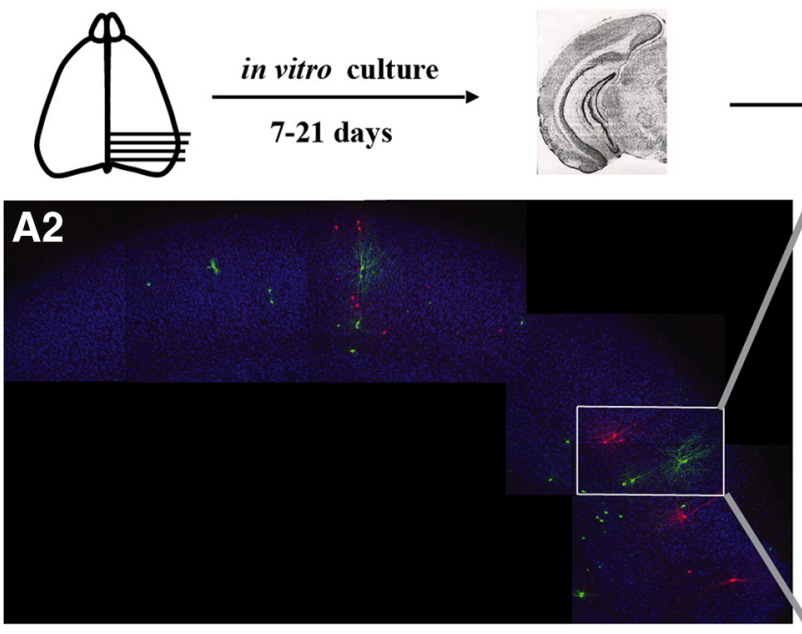

Visual cortical slices

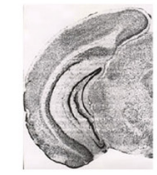

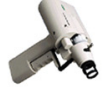

gene gun transfection

$$
>2 \text { days }
$$

\section{Confocal imaging}
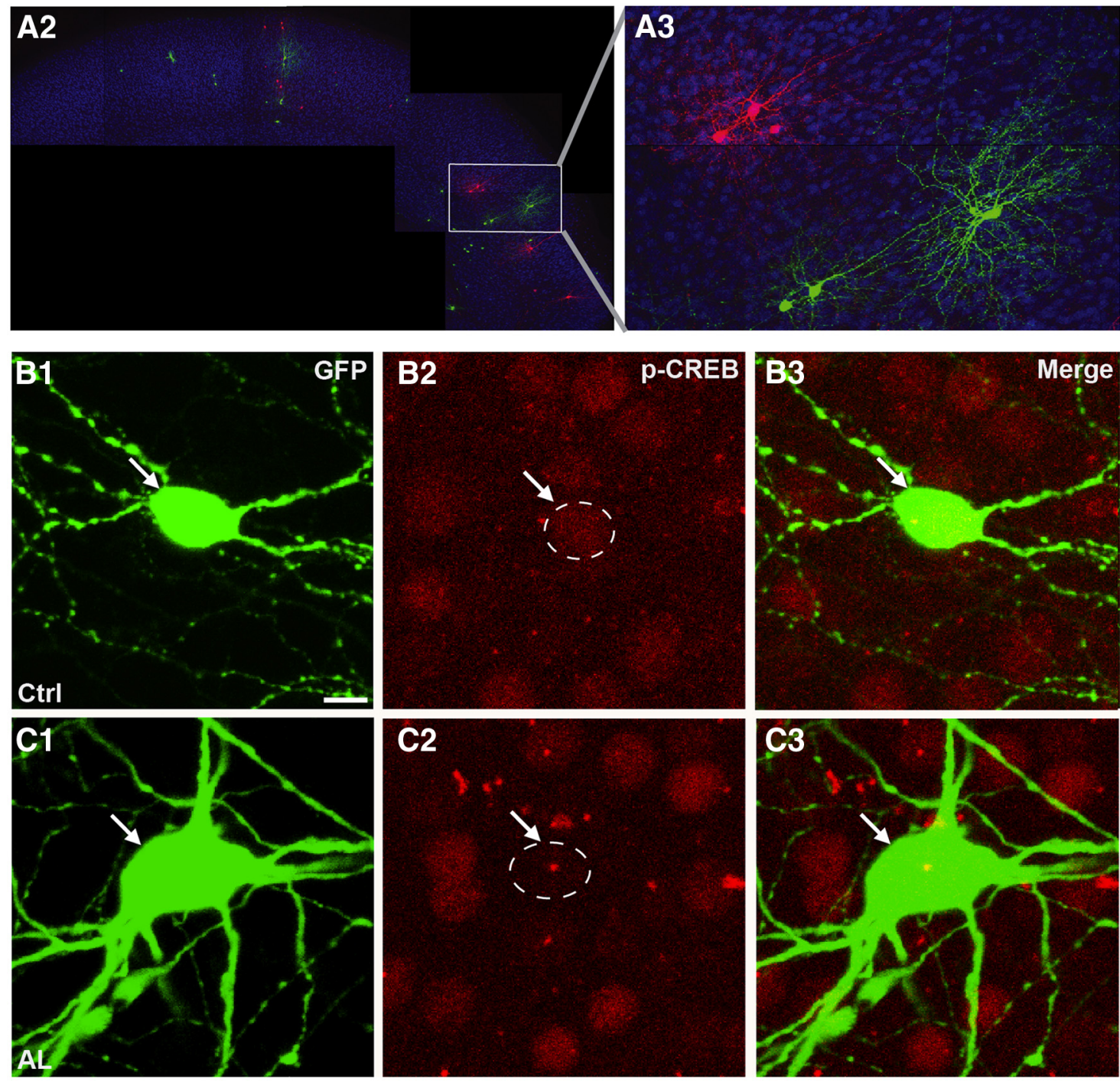

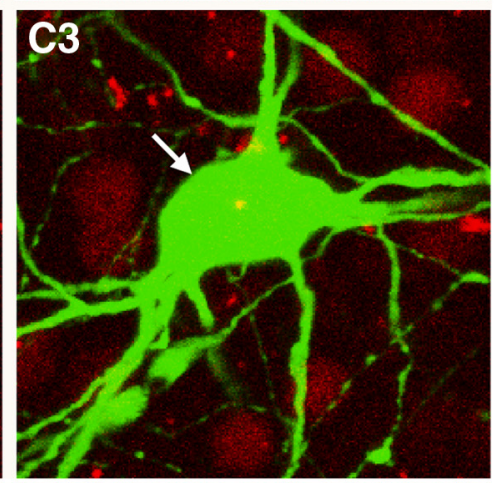

Figure 1. Activation of AlstR reduces activity-dependent increase of $p C R E B$ expression. $A$, Schematics of experimental procedure. Cortical organotypic cultures were biolistically transfected with $\mathrm{P}_{667}-\mathrm{GFP} / \mathrm{P}_{667}$-AlstR and $\mathrm{P}_{667}$-tdTomato to colabel allatostatin-expressing cells in green and control basket cells in red in the same organotypic slice. $B, C, \mathrm{P}_{667}$-GFP/P $\mathrm{P}_{667}$-AlstR transfected basket cells treated with allatostatin $(\boldsymbol{C})$ show decreased nuclear pCREB immunoreactivity $(\mathbf{C}$, arrow) compared with untransfected neighboring cells, whereas pCREB levels in untreated, AlstR-transfected basket cells $(\boldsymbol{B})$ do not differ from neighboring cells (B2, arrow). Scale bar, $10 \mu \mathrm{m}$.

ket cells (Fig. 2B, green) treated with allatostatin from EP17-24 formed perisomatic innervation characterized by multiple terminal axon branches and clustered boutons that were indistinguishable from allatostatin-treated control cells (Fig. 2A, red; Fig. 2E, F,H; boutons/soma $\pm \mathrm{SEM}=9.4 \pm 0.2$ for control versus $9.4 \pm 0.4$ for AlstR-cells, one-way ANOVA, Tukey's test, $p>0.05$; bouton size \pm $\mathrm{SEM}=0.98 \pm 0.03 \mu \mathrm{m}$ for control cells versus $1.00 \pm 0.04 \mu \mathrm{m}$ for AlstR-expressing cells, one-way ANOVA, Holm-Sidak's test, $p>$ $0.05)$. However, when comparing the fraction of innervated cells over the total number of potential targets in a confocal stack, we found a significant reduction of the percentage of innervated cells by each basket cell expressing AlstR as compared with controls in the same allatostatin-treated slice (Fig. 2G; $51 \pm 3 \%$ and $69 \pm 3 \%$, respectively, one-way ANOVA, Holm-Sidak's test, $p<0.05)$. To quantify perisomatic innervation, we imaged the basket cell axon in the first $150 \mu \mathrm{m}$ from the parent soma, because in this region we found that the characteristics of the innervation are reproducible across basket cells. By quantifying innervation percentage in these axonal fields, however, we might have introduced a bias toward highly innervated regions. Therefore, we next fully reconstructed basket axon arbors along with all the pyramidal cell somata, and we found similar results (Fig. 3; $50 \pm 3 \%$ for AlstR-cells vs $67 \pm 1 \%$ for controls, $t$ test, $p<0.001)$. Because basket cell axons also innervate the proximal dendrites of pyramidal cells, which were not detected in our analysis, the fraction of innervated pyramidal cells might be somewhat underestimated. On the other hand, such underestima- 

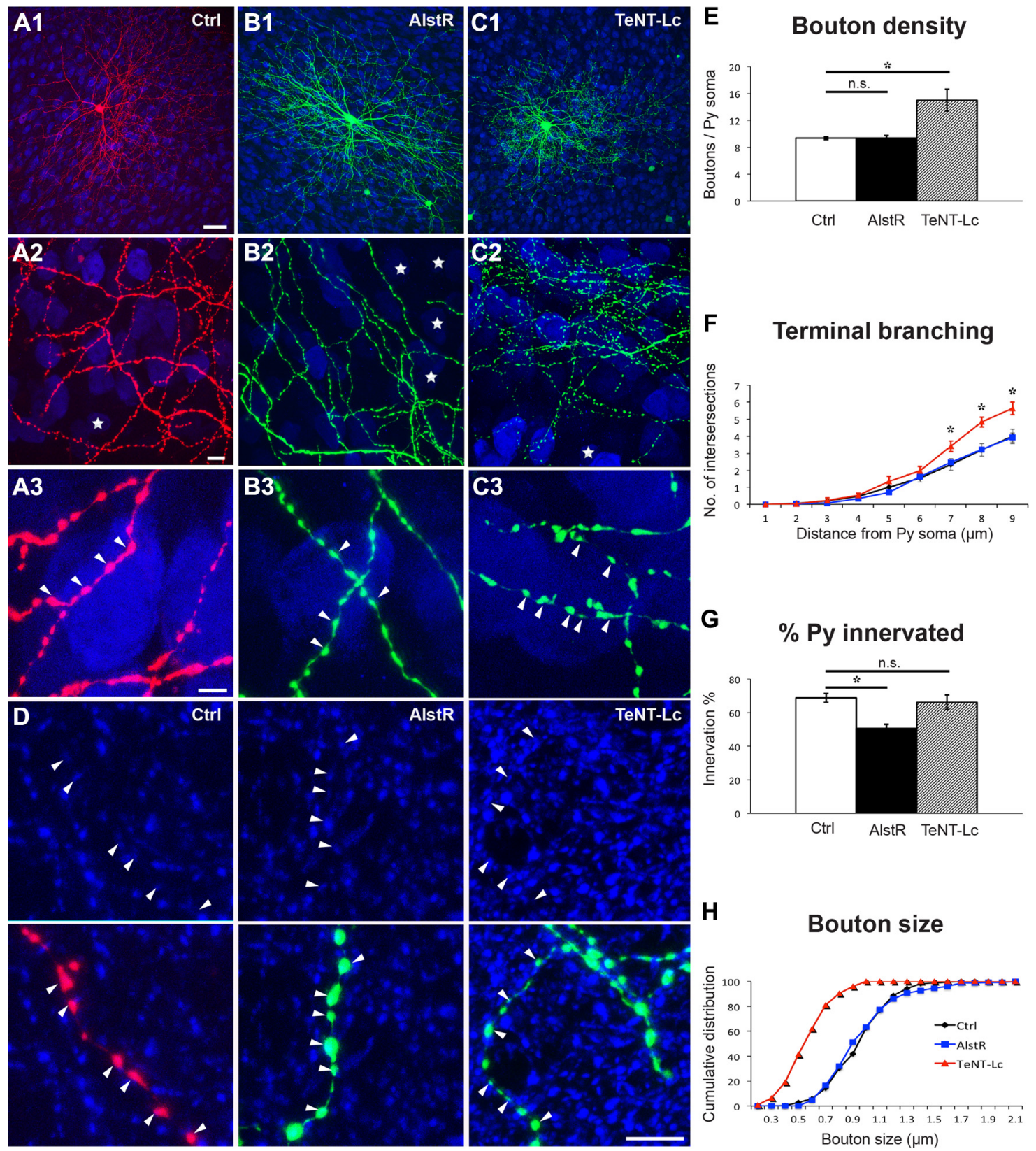

Figure 2. Reducing basket cell excitability and suppressing neurotransmitter release have opposite effects during the peak of perisomatic synapse maturation. $A$, Control basket cell (Ctrl, red) at EP24 with exuberant innervation field characterized by extensive branching contacting the majority of potential targets, dense boutons along axons (A2), and terminal branches with prominent and clustered boutons ( $\boldsymbol{A} \mathbf{3}$, arrowheads) around pyramidal cell somata (NeuN immunostaining, blue). Stars indicate pyramidal cell somata that are not innervated in the projected confocal stack. $\boldsymbol{B}$, AlstR-expressing basket cell (green) treated with allatostatin from EP17-24 shows overall similar axon size and morphology (B1), however, it is easier to find noninnervated targets in the axonal arbor territory (B2, stars). Terminal branching, bouton size, and density $(\boldsymbol{B} 3)$ around innervated pyramidal cell somata appear similar to those from control cells. $\boldsymbol{C}$, TeNT-Lc-expressing basket cell (green) shows overall similar axon size (C1). The fraction of innervated targets does not appear affected (C2), however, perisomatic innervations are characterized by more numerous, smallerlooking boutons (C3, arrowheads) compared with Ctrl cells. $\mathbf{A 3}, \mathbf{B 3}$, and $\mathbf{C}$ are from regions in A2, B2, and C2. Scale bars (in A1) A1-C1,50 $\mu \mathrm{m}$; (in A2) A2-C2, $10 \mu \mathrm{m}$; (in A3) A3-C3,5 $\mu \mathrm{m}$. D, GFP or RFP-positive boutons colocalize with GAD65 (blue) in controls, AlstR or TeNT-Lc basket cells, suggesting that even the smaller boutons in TeNT-Lc-transfected cells express GABAergic synaptic markers. Scale bar, $5 \mu \mathrm{m}$. $\boldsymbol{E}, \boldsymbol{F}$, AlstR-expressing cells show no differences in bouton density $(\boldsymbol{E})$ and terminal branching $(\boldsymbol{F})$ compared with aged-matched controls $(p>0.05$, n.s.), whereas TeNT-Lc-expressing cells show a statistically significant increase of both parameters $\left({ }^{*} p<0.05\right) ; n=67$ perisomatic innervations from 6 control basket cells for controls, 58 innervations from 6 AlstR basket cells, and 60 perisomatic innervations from 6 TeNT-Lc basket cells. G, At EP24, the fraction of potentially innervated targeted neurons is not affected for TeNT-Lc-expressing cells, but it is significantly reduced for AlstR-transfected cells $\left({ }^{*} p<0.05\right) ; n=6$ basket cells for all experimental groups. $\boldsymbol{H}$, Bouton size is significantly reduced in TeNT-Lc-transfected cells compared with controls and AlstR-expressing cells (Kolmogorov-Smirnov (K-S) test, $p<0.001$ ); $n=150$ boutons from 6 basket cells for each group. Values in $\boldsymbol{D}-\boldsymbol{F}$ represent mean \pm SEM.

tion, if it exists, should equally affect control and AlstR-expressing cells. Few cells were transfected in each cortical slice (2-10/slice), thus alterations of global neural activity cannot be responsible for the observed effects. Together, these results suggest that reduction of basket cell excitability may affect the number of potential targets contacted by basket cells without affecting the extent of perisomatic innervation occurring on contacted cells.

To suppress neurotransmitter release, we used TeNT-Lc fused with EGFP, which has been shown to efficiently block both evoked and spontaneous neurotransmitter release in dissociated 
A
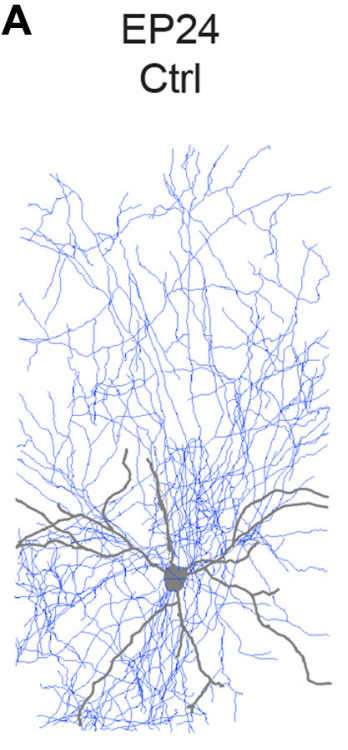

EP24

AlstR

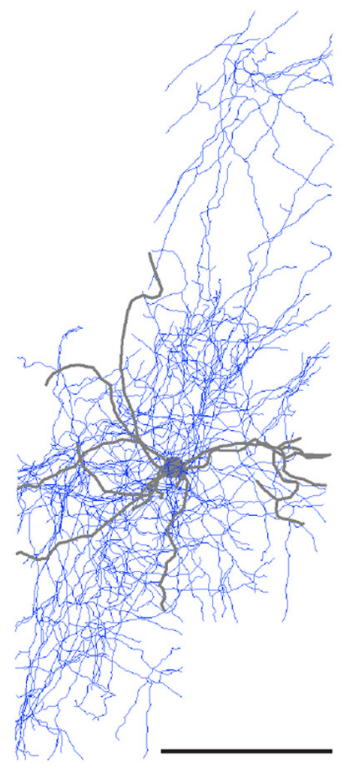

EP32

Ctrl

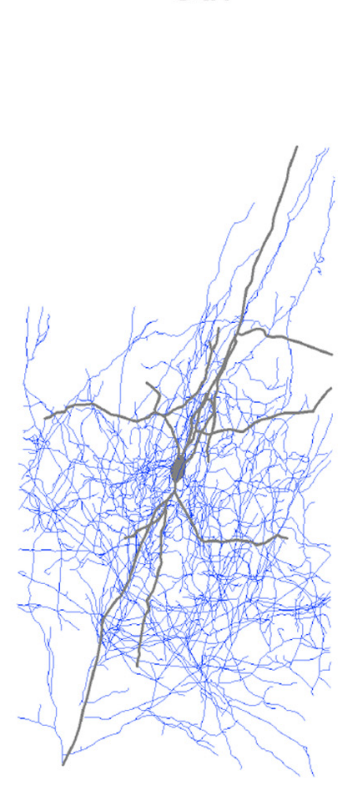

EP32 AlstR

\section{B}
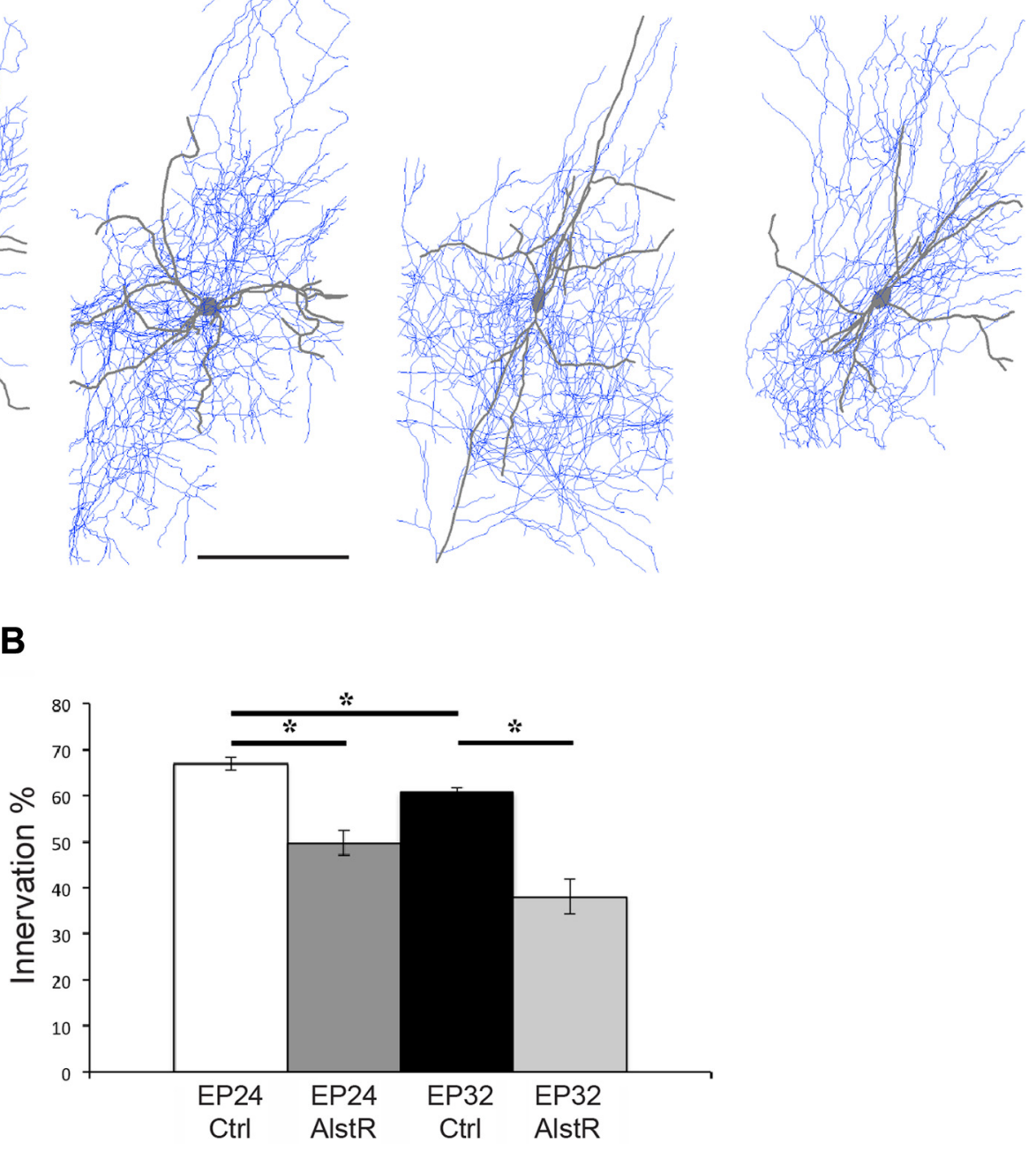

Figure 3. Basket cell axon analysis suggests the occurrence of pruning after the peak of perisomatic synapse proliferation. $\boldsymbol{A}$, Examples of fully reconstructed basket cells. Scale bar, $150 \mu \mathrm{m}$. $\boldsymbol{B}$, Full axon reconstruction of control basket cells at EP24 and EP32 show that the percentage of potentially innervated cells is significantly smaller at EP32 compared with EP24 ( ${ }^{*} p<0.05, n=5$ for both ages). In addition, AlstR activation from EP17-24 ( $n=3)$ and from EP26-32 $(n=3)$ significantly reduces the fraction of innervated cells $\left({ }^{*} p<0.05\right)$, consistent with what was shown by quantifying the innervation percentage in confocal stacks acquired in the first $150 \mu \mathrm{m}$ from the basket cell soma (Fig. $2 \mathrm{G}$ ).

hippocampal neurons (Ben Fredj et al., 2010). This manipulation induced completely different effects from those observed with AlstR. Basket cells expressing TeNT-Lc from EP17-24 were characterized by denser axonal arbors and an increased number of smaller, homogenous boutons around the innervated somata compared with control cells (Fig. 2A,C). Quantitative analysis showed that terminal branching and perisomatic bouton density were indeed significantly increased (Fig. 2E, F; boutons/soma \pm $\mathrm{SEM}=9.4 \pm 0.2$ for controls versus $15.0 \pm 1.6$ for TeNT-Lcexpressing cells, one-way ANOVA, Tukey's test, $p<0.05$ ) and bouton size reduced (Fig. $2 \mathrm{H}$; bouton size $\pm \mathrm{SEM}=0.98 \pm$ $0.03 \mu \mathrm{m}$ for controls versus $0.60 \pm 0.03 \mu \mathrm{m}$ for TeNT-Lccells, one-way ANOVA, Holm-Sidak's test, $p<0.001$ ). Axon density was also significantly increased in TeNT-Lc-cells compared with control basket cells (axon length $(\mu \mathrm{m}) /$ volume $\left(\mu \mathrm{m}^{3}\right)\left({ }^{*} 10^{-3}\right) \pm \mathrm{SEM}=9 \pm 2$ for TeNT-Lc-cells vs $5.7 \pm 0.4$ and $4.7 \pm 0.6$ for AlstR and control cells, respectively, $n=5$ for control and $n=3$ for TeNT-Lc and AlstR cells, one-way ANOVA $p<0.05)$. On the other hand, the average total bouton density per field was not statistically different in TeNT-Lcexpressing basket cells compared with AlstR-expressing and control cells (bouton $/ 10 \mu \mathrm{m}$ axon length $\pm \mathrm{SEM}=4.1 \pm 0.2$ for controls vs $4.4 \pm 0.2$ and $4.5 \pm 0.6$ for AlstR and TeNT-Lc cells, one-way ANOVA $p>0.05$ ). The vast majority of GFPlabeled boutons in basket cells expressing TeNT-Lc colocalized with GAD65, a presynaptic GABAergic marker (Fig. 2D), thus suggesting a size reduction of synaptic contacts caused by the suppression of neurotransmission. Conversely, the percentage of innervated somata was not significantly affected (Fig. 2G), suggesting that the complete blockade of GABA release might affect local synapse and branches dynamics (the balance between elimination and addition of new synapses and branches) without changing the number of target cells contacted by the basket cell. 
Next, we investigated whether these two manipulations could affect basket cell innervation after the peak of perisomatic bouton formation, from EP26 to EP32. Similar to what we observed during the maturation phase, reducing neural excitability in single basket interneurons by allatostatin-mediated AlstR activation did not affect bouton density, terminal branching, or bouton size (Fig. $4 A, B, D, E, G$; boutons/soma $\pm \mathrm{SEM}=11.1 \pm 0.8$ for controls vs $11.0 \pm 0.3$ for AlstR-cells; bouton size $\pm \mathrm{SEM}=1.02 \pm$ $0.02 \mu \mathrm{m}$ for control vs $1.01 \pm 0.01$ for AlstR-cells; one-way ANOVA, Holm-Sidak's test, $p>0.05$ ), while it significantly reduced the percentage of targeted neural somata (quantified in axonal fields $<150 \mu \mathrm{m}$ from the basket cell soma) as compared with controls (Fig. $4 F ; 70 \pm 3 \%$ for controls vs $50 \pm 3 \%$ for AlstR-cells; one-way ANOVA, Holm-Sidak's test, $p<0.001$ ). Surprisingly, suppressing neurotransmitter release in this time window caused completely different effects as compared with the earlier phase. TeNT-Lc transfected basket cells formed perisomatic innervation with fewer terminal axon branches and fewer, irregular-sized boutons around innervated somata compared with control cells (Fig. 4A,C). Quantitative analysis showed significantly reduced axonal branching, bouton density (Fig. $4 D-E$; boutons/soma $\pm \mathrm{SEM}=11.1 \pm 0.8$ for control vs $5.11 \pm 0.4$ for TeNT-Lc cells; one-way ANOVA, Holm-Sidak's test, $p<0.05$ ), and perisomatic bouton sizes (Fig. $4 G$, mean bouton size \pm $\mathrm{SEM}=1.02 \pm 0.02 \mu \mathrm{m}$ for controls vs $0.76 \pm 0.05 \mu \mathrm{m}$ for TeNT-Lc cells; one-way ANOVA, Holm-Sidak's test, $p<0.05$ ). In addition, suppressing neurotransmitter release caused an even more drastic reduction in percentage of innervated cells as compared with allatostatin treatment (Fig. $4 F ; 70 \pm 3 \%$ for controls, $50 \pm 3 \%$ for AlstR cells, and $39 \pm 4 \%$ for TeNT-Lc cells; one-way ANOVA, Holm-Sidak's test, $p<0.001)$. Axon density was significantly decreased in both TeNT-Lc- and AlstR-expressing cells compared with control basket cells (axon length $(\mu \mathrm{m}) /$ volume $\left(\mu \mathrm{m}^{3}\right)\left({ }^{\star} 10^{-3}\right) \pm \mathrm{SEM}=9.6 \pm 0.6$ for controls vs $6 \pm 2$ and $4.5 \pm 0.5$ for AlstR and TeNT-Lc cells, respectively; $n=5$ for control, $n=4$ for AlstR, and $n=3$ for TeNT-Lc cells, one-way ANOVA, $p<0.05)$, while average total bouton density per field was invariant (bouton $/ 10 \mu \mathrm{m}$ axon length $\pm \mathrm{SEM}=2.7 \pm 0.5$ for controls vs $2.6 \pm 0.6$ and $3.7 \pm 0.6$ for AlstR- and TeNT-Lcexpressing cells, one-way ANOVA, $p>0.05$ ). After EP26, the number of perisomatic boutons and the extent of terminal branching are stable overall (Chattopadhyaya et al., 2004, 2007). By analyzing completely reconstructed basket cell axon arbors, however, we found that the percentage of contacted cells decreased slightly but significantly from EP26 to EP32 (Fig. 3, $67 \pm$ $1 \%$ for EP24 and $61 \pm 1 \%$ for EP32 basket cells, $t$ test, $p<0.05$ ), suggesting an ongoing pruning process occurring during this period.

All together, these results indicate that basket cell excitability and neurotransmitter release play distinct and specific roles during the different stages of the establishment of the basket cell's innervation field.

\section{Discussion}

Activity guides the patterning of synaptic connections in the developing nervous system. Specifically, differences in the activity of converging inputs are thought to cause elimination of synapses from less active inputs and increase connectivity with more active inputs. This is supported by numerous studies in the visual and neuromuscular systems (Buffelli et al., 2003; Ruthazer et al., 2003; Huberman et al., 2008). However, recent results have challenged this notion. Studies of RGC axons in the zebrafish tectum showed that activity could regulate branch addition and stability rather than retraction (Hua et al., 2005; Ben Fredj et al., 2010). A second study (Kerschensteiner et al., 2009) showed that rather than engaging in competition, the axons of ON and OFF bipolar cells refine their connectivity with RGCs independent of one another even when they converge stably onto a single postsynaptic cell. Therefore, even for excitatory synapses, there appears to be different mechanisms for the role of activity in circuit refinement. This makes it even harder to predict how neural activity might modulate GABAergic innervation, which is characterized by a dense, local axon arbor, as opposed to projecting inputs, and involves an inhibitory neurotransmitter.

As key mediators of neural activity, neurotransmitters are particularly well placed to couple the activation state of a cell with synaptic morphogenesis and refinement. A previous study from Chattopadhyaya et al. (2007) showed that single basket cell knockdown of Gad1, which encodes for GAD67, the enzyme responsible for the majority of GABA synthesis ( $~ 90 \%$; Pinal and Tobin, 1998) during the maturation of perisomatic innervation, reduced both perisomatic bouton density and the number of cells innervated by the affected basket cell. Gad1 knock-out strongly reduces but does not completely eliminate GABA synthesis, as GAD65, the other GABA-synthesizing enzyme, is still expressed in the basket cell. In this study, we used two different approaches to modulate neurotransmission: allatostatin-mediated activation of AlstR to reduce neuron excitability and action potential firing, which in turn reduces neurotransmitter release (Birgül et al., 1999), and TeNT-Lc expression to suppress both stimulusevoked and spontaneous release of synaptic vesicles (Ben Fredj et al., 2010). Our results show that reducing levels of neurotransmission decreased the number of targeted postsynaptic cells by a single basket cell in both of the developmental time windows studied, namely, during and after the maturation of perisomatic innervation, while suppressing neural transmission had opposing effects depending on the developmental stage of the basket cell.

During the maturation phase of perisomatic GABAergic innervation, a minimal level of GABA release seems to be necessary to engage the mechanisms that select nascent contacts, based most likely on the correctness of the postsynaptic target or on the relative synaptic strength compared with neighboring synaptic contacts. The idea that neurotransmitters inhibit synapse formation has a precedent in neuromuscular junction formation, where evidence shows that ACh destabilizes nascent postsynaptic sites by dispersing postsynaptic receptors, and that one major physiological role of agrin is to counteract this antisynaptogenic influence (Misgeld et al., 2005). TeNT-Lc-expressing basket cells from EP17-24 show a normal innervation pattern in terms of the number of innervated cells, however, they form more synapses and terminal branches around each innervated neuron. This result is consistent with the hypothesis that GABAergic transmission is not necessary for targeted selection (Huang et al., 2007) but plays a role in synapse validation and stabilization.

It has been suggested that in the hippocampus, a single pyramidal cell may receive perisomatic inputs from 5 to 7 basket cells. It is possible that different basket cells compete for the control of a target cell, with neurotransmission serving as a natural indicator of the activity of the basket cells and of the stronger/more efficient synapses. A basket interneuron that is less capable of eliciting a response from the target than the neighbor basket cells, as in the case of AlstRexpressing basket cells, may start to lose targets but will still form normal perisomatic innervation on the targets that are retained. If GABA transmission is even more drastically reduced, as in Gad1 knock-out cells, the basket cell will start to lose territory and form 

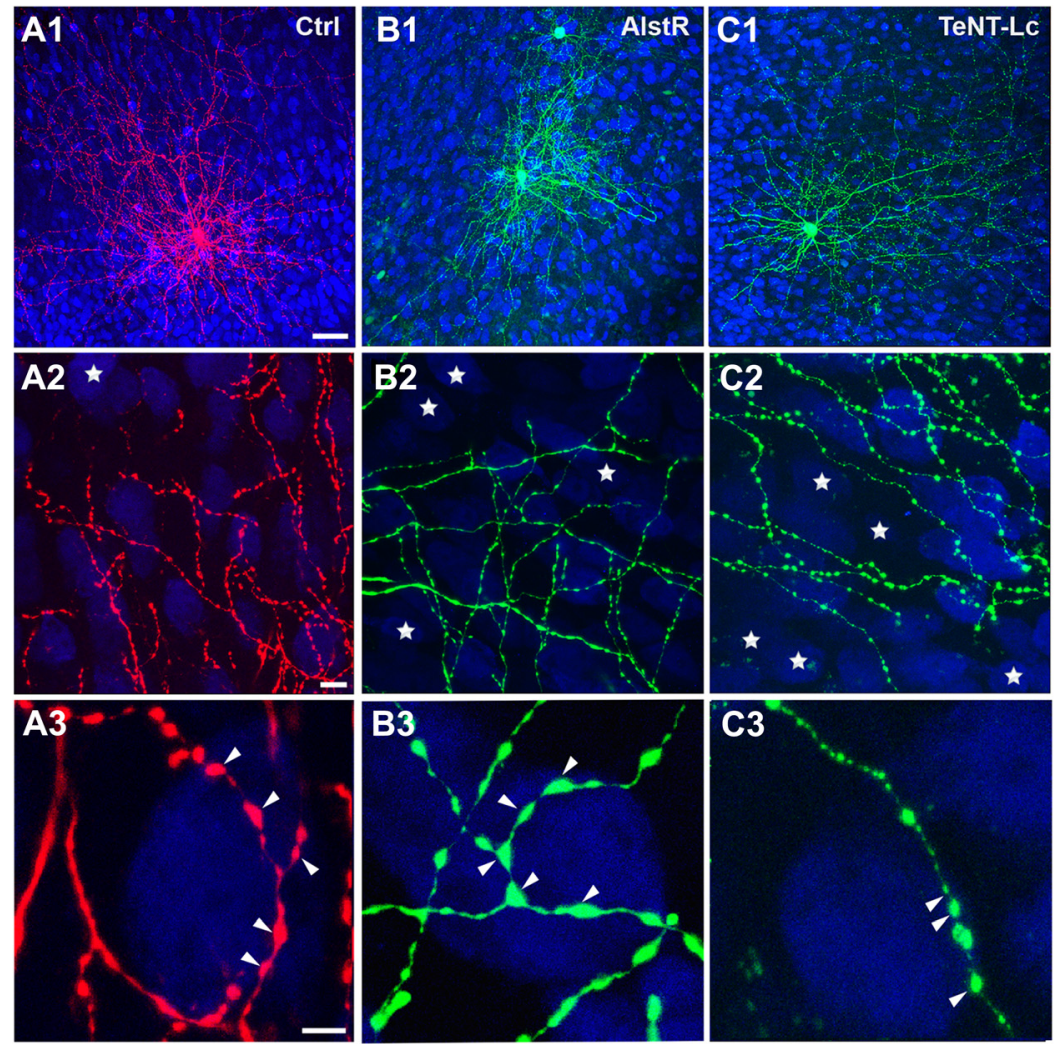

D

Bouton density

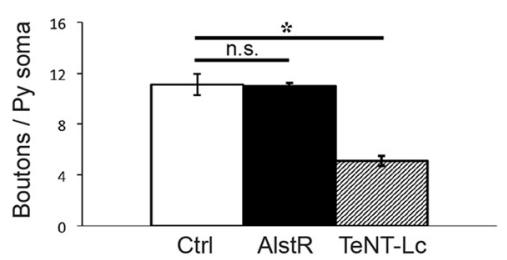

E

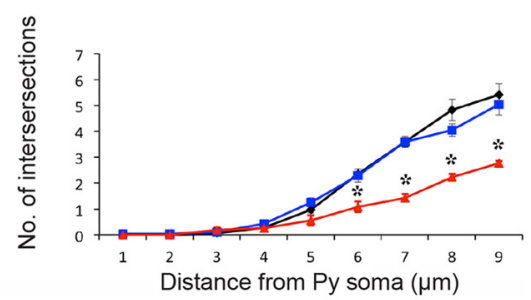

$\mathbf{F}$

$\%$ Py innervated



$\mathbf{G}$

Bouton size

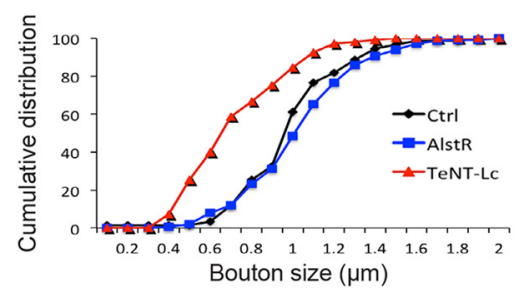

Figure 4. Suppressing neurotransmitter release after the peak of perisomatic synapse maturation induces a more severe loss of perisomatic innervations than reducing neural excitability. A-C, Activation of AlstR from EP26-32 does not affect terminal branching or perisomatic boutons formed by AlstR-expressing cells ( $\boldsymbol{B} 3$, arrowheads) compared with controls $(\boldsymbol{A} 3)$, but it diminishes the number of potentially innervated postsynaptic cells ( $\boldsymbol{A} 2, \boldsymbol{B 2}$; stars represent noninnervated pyramidal somata in the projected confocal stack). On the other hand, TeNT-Lc-expressing cells show a reduction both in percentage of innervated cells $(\boldsymbol{C} 2)$ and perisomatic innervation $(\mathbf{C})$. Boutons appear more irregular with some large and many smaller ones (arrowheads). $\boldsymbol{A} \mathbf{3}, \boldsymbol{B} \mathbf{3}$, and $\mathbf{C}$ are from regions in $\boldsymbol{A 2}, \mathbf{B} 2$, and $\boldsymbol{C}$. Scale bars: (in A1) A1-C1,50 $\mu \mathrm{m}$; (in A2) A2-C2, $10 \mu \mathrm{m}$; (in A3) A3-C3,5 $\mu \mathrm{m}$. D, E, Quantification shows that terminal branching and perisomatic bouton density is significantly affected only in TeNT-Lc-transfected cells, but not by AlstR activation ( $\left.{ }^{*} p<0.001\right) ; n=60$ perisomatic innervations from 6 basket cells for each experimental group. $F$, At EP32, both AlstR- and TeNT-Lc-expressing cells show reduced innervation of pyramidal somata compared with control $\left({ }^{*} p<0.001\right) ; n=6$ basket cells for each group. $G$, AlstR-expressing cells havenormal-sized boutons compared with controls, but TeNT-Lc-expressing cells show a significant reduction in bouton size (K-Stest, $p<$ $0.001) ; n=6$ basket cells and 150 boutons. Values in $\boldsymbol{D}-\boldsymbol{F}$ represent mean \pm SEM.

only smaller and fewer synapses on the pyramidal cells that it still innervates. Our observations are consistent with what has been shown for the neuromuscular junction, where interaxonal differences in the ability of an axon to activate a muscle fiber can account for the outcome of synaptic competition (Buffelli et al., 2003; Kasthuri and Lichtman, 2003), therefore suggesting that local cortical interneurons might follow similar rules for the control of the axonal arbor territory as projection neurons.

One caveat is that AlstR activation induces hyperpolarization of the entire neuron while TeNT-Lc does not, which could differentially affect gene expression by the basket cell and in turn its ability to form or eliminate synapses. Basket cells transfected with both AlstR and TeNT-Lc and treated with allatostatin from EP17 to EP24 form perisomatic innervations indistinguishable from those formed by basket cells transfected with TeNT-Lc only (our unpublished observation), suggesting that reduced cell excitability does not influence the ability of a basket cell to form supranumerary synapses following the suppression of neurotransmitter release. However, we cannot rule out that presynaptic factors other than neurotransmitter release levels play a role on the effects observed following AlstR activation.

Further, Ben Fredj et al. (2010) showed that TeNT-Lc expression completely suppressed evoked neurotransmitter release and reduced, but did not completely suppress, spontaneous fusion of synaptic vesicles; thus, a low level of spontaneous release of GABA may still occur. It is also possible that TeNT-Lc might block fusion of vesicles carrying signals other than neurotransmitters. In a recent study, Huang and collaborators (Wu et al., 2012) completely blocked GABA release in single basket cells during the peak of perisomatic synapse proliferation, either by simultaneously removing GAD67 and GAD65 or by removing the GABA vesicular transporter vGAT, and found increased density and reduction in size of the boutons formed by basket cell axons, consistent with our results. Thus, it is likely that the majority, if not all, of the effects we observed in TeNT-Lc-expressing cells are mainly due to the suppression of GABA release.

In summary, we found that the establishment of a mature basket axonal arbor territory occurs in two phases. During the peak of perisomatic synapse proliferation, neurotransmitter release plays the dual role of promoting both synapse elimination and synapse maturation. It is possible that GABAergic synaptic transmission produces two signals, a "protective/rewarding" signal and an "elimination/punishing" signal. A less active synapse would have reduced production of these signals, and thus, in the absence of protection, would be susceptible to the elimination signal from neighboring, more active synaptic sites. On the other hand, 
suppression of neurotransmission would also eliminate the punishing signal and explain the supranumerary synapses formed by basket cells expressing TeNT-Lc. A similar model has been suggested to take place during the process of activity-dependent axon elimination at the neuromuscular junction (Lichtman and Colman, 2000). It is interesting to note that GABA release from neighboring, untransfected basket cells seems unable to rescue the effects of TeNT-Lc expression, suggesting that the punishing signal might have a direct paracrine effect on the basket cell axon. Conversely, the production of and/or ability to respond to the reward signal might depend on the relative activity levels of neighboring basket cells. Identifying the molecular identities of the rewarding and punishing signals activated by GABA release is the key step to shed light on the cellular mechanisms involved during this developmental phase.

After this phase, and in a relatively short time, basket cell response to suppressed neurotransmission changes, and the maintenance of its innervation field becomes exquisitely dependent on neurotransmission levels, as supported by the fact that TeNT-Lc-expressing cells show a more severe phenotype as compared with AlstR-transfected ones. The molecular mechanisms underlying this switch are currently unknown, but it could involve either presynaptic changes (for example, in the transcriptional profile of the basket cells) and/or changes in pyramidal cells and glia-derived factors, which could retrogradely modulate GABAergic axons.

Taken together, our results show for the first time that neural activity and neurotransmission function in the refinement of GABAergic basket cell innervation follows different rules at different developmental stages. Selective modulation of inhibitory networks is currently being investigated as a tool to reintroduce plasticity, and, therefore, facilitate the restoration of normal function to the diseased brain (Southwell et al., 2010). A better comprehension of the molecular mechanisms regulating GABAergic innervation might aid these efforts.

\section{References}

Ango F, di Cristo G, Higashiyama H, Bennett V, Wu P, Huang ZJ (2004) Ankyrin-based subcellular gradient of neurofascin, an immunoglobulin family protein, directs GABAergic innervation at purkinje axon initial segment. Cell 119:257-272.

Ango F, Wu C, Van der Want JJ, Wu P, Schachner M, Huang ZJ (2008) Bergmann glia and the recognition molecule CHL1 organize GABAergic axons and direct innervation of Purkinje cell dendrites. PLoS Biol 6(4):e103.

Ben Fredj N, Hammond S, Otsuna H, Chien CB, Burrone J, Meyer MP (2010) Synaptic activity and activity-dependent competition regulates axon arbor maturation, growth arrest, and territory in the retinotectal projection. J Neurosci 30:10939-10951.

Birgül N, Weise C, Kreienkamp HJ, Richter D (1999) Reverse physiology in drosophila: identification of a novel allatostatin-like neuropeptide and its cognate receptor structurally related to the mammalian somatostatin/ galanin/opioid receptor family. EMBO J 18:5892-5900.

Bosman LW, Konnerth A (2009) Activity-dependent plasticity of developing climbing fiber-Purkinje cell synapses. Neuroscience 162:612-623.

Buffelli M, Burgess RW, Feng G, Lobe CG, Lichtman JW, Sanes JR (2003) Genetic evidence that relative synaptic efficacy biases the outcome of synaptic competition. Nature 424:430-434.

Chattopadhyaya B, Di Cristo G, Higashiyama H, Knott GW, Kuhlman SJ, Welker E, Huang ZJ (2004) Experience and activity-dependent maturation of perisomatic GABAergic innervation in primary visual cortex during a postnatal critical period. J Neurosci 24:9598-9611.

Chattopadhyaya B, Di Cristo G, Wu CZ, Knott G, Kuhlman S, Fu Y, Palmiter RD, Huang ZJ (2007) GAD67-mediated GABA synthesis and signaling regulate inhibitory synaptic innervation in the visual cortex. Neuron 54:889-903.

Di Cristo G, Chattopadhyaya B, Kuhlman SJ, Fu Y, Bélanger MC, Wu CZ, Rutishauser U, Maffei L, Huang ZJ (2007) Activity-dependent PSA expression promotes the maturation of GABA inhibition and the onset of critical period plasticity. Nat Neurosci 10:1569-1577.

Hua JY, Smear MC, Baier H, Smith SJ (2005) Regulation of axon growth in vivo by activity-based competition. Nature 434:1022-1026.

Huang ZJ, Di Cristo G, Ango F (2007) Development of GABA innervation in cerebral and cerebellar cortex. Nat Rev Neurosci 8:673-686.

Huberman AD, Feller MB, Chapman B (2008) Mechanisms underlying development of visual maps and receptive fields. Annu Rev Neurosci 31:479-509.

Kasthuri N, Lichtman JW (2003) The role of neural identity in synaptic competition. Nature 424:426-430.

Kerschensteiner D, Morgan JL, Parker ED, Lewis RM, Wong RO (2009) Neurotransmission selectively regulates synapse formation in parallel circuits in vivo. Nature 460:1016-1020.

Lichtman JW, Colman H (2000) Synapse elimination and indelible memory. Neuron 25:269-278.

Marina N, Abdala AP, Trapp S, Li A, Nattie EE, Hewinson J, Smith JC, Paton JF, Gourine AV (2010) Essential role of Phox2b-expressing ventrolateral brainstem neurons in the chemosensory control of inspiration and expiration. J Neurosci 30:12466-12473.

Misgeld T, Kummer TT, Lichtman JW, Sanes JR (2005) Agrin promotes synaptic differentiation by counteracting an inhibitory effect of neurotransmitter. Proc Natl Acad Sci U S A 102:11088-11093.

Pinal CS, Tobin AJ (1998) Uniqueness and redundancy in GABA production. Perspect Dev Neurobiol 5:109-118.

Rossignol E (2011) Genetics and function of neocortical GABAergic interneurons in neurodevelopmental disorders. Neural Plast 2011, 2011:649325.

Ruthazer ES, Akerman CJ, Cline HT (2003) Control of axon branch dynamics by correlated activity. Science 301:66-70.

Southwell DG, Froemke RC, Alvarez-Buylla A, Stryker MP, Gandhi SP (2010) Cortical plasticity induced by inhibitory neuron transplantation. Science 327:1145-1148

Wu X, Fu Y, Knott G, Lu J, Di Cristo G, Huang ZJ (2012) GABA signaling promotes synapse elimination and axon pruning in developing cortical inhibitory interneurons. J Neurosci, in press.

Zhou Y, Won J, Karlsson MG, Zhou M, Rogerson T, Balaji J, Neve R, Poirazi P, Silva AJ (2009) CREB regulates excitability and the allocation of memory to subsets of neurons in the amygdala. Nat Neurosci 12:14381443. 